\title{
Application of hall for workshop of meta- synthetic engineering in the knowledge consulting services
}

\author{
Feng LanPing \\ School of Enterprise Management, HoHai University \\ Changzhou, China \\ Fenglp@hhuc.edu.cn
}

\author{
Dong ZhiHui \\ School of Business, HoHai University \\ Nanjing, China \\ dong9650@126.com
}

\begin{abstract}
Aiming at the lack of expert and user participation problems in knowledge consulting, the paper proposes knowledge consulting service based on Hall for workshop of meta-synthetic engineering, and its characteristics, model and analysis of key technology are analyzed in detail, knowledge map provides the knowledge sharing platform of tacit and explicit knowledge, and also improve the process of visualization. In the model the expert and user are introduced and take part in discussion. In discussion, experts have a large number of tacit knowledge in their mind, users can ensure the accuracy and efficiency of the discussion, furthermore, the participation of users and experts can promote the formation of collective intelligence. The model can be applied to any complex knowledge consulting system, and can also improve the expert participating in knowledge sharing. At last a case study show the frame can improve the efficiency of knowledge consulting and meet the users need.
\end{abstract}

Keywords-Knowledge Consulting; knowledge map; visuali zation; Hall for workshop of meta-synthetic engineering; $\mathrm{HW}$ ME; knowledge retrieval;

\section{INTRODUCTION}

Knowledge consulting is guided by the user needs, knowledge interaction between knowledge consulter and users as a precondition to achieve value-added services, because of its services and methods to better meet the needs of users than traditional reference service, gradually become a modern reference way to adapt to the user knowledge needs in knowledge economy era. Wang TianNi proposed that knowledge consulting is the growth points of library knowledge services in the era of the big data ${ }^{[1]}$; Ma Tianshu proposed that knowledge consulting model is divided into knowledge expert mode, the project team and process mapping three main modes according to user needs change ${ }^{[2]}$. Zeng DeYun starting from the integrating library resources and user-centered, knowledge consulting modes can be divided into one-stop, interactive network services and knowledge transfer service mode three main modes ${ }^{[3]}$.Li WENHUI based on the experience of knowledge consultation by the Unite and Combined Digital Reference Service system, and explains the meaning, characteristics, carrying out conditions and work flows of knowledge consultation, the main process of knowledge consulting can be divided into recognition analysis, collection restructure, knowledge production and evaluation feedback four stages ${ }^{[4]}$. Liu
Qing in Japan collaborative reference database example from knowledge creation spiral theory to build collaborative reference service system ${ }^{[5]}$.In knowledge consulting, experts can be divided into reality experts and virtual experts, reality expert has a lot of knowledge and experience stored in their minds, the virtual expert knowledge refers to the summary and the reuse explicit knowledge of produced in the discussion process, without the participation of experts will be difficult to play the biggest role in the extent of its knowledge share and innovation, user participating in discussions not only can improve the efficiency of discussion, it can also guarantee the correctness of discussion, for the use of expert knowledge collective intelligence and user participate in consultation discussion is still relatively little, the study of consulting process visualization are rarely.

Hall for workshop of meta-synthetic engineering (HWME) was widely used to solve complex problems since it was proposed, knowledge consulting is including users, knowledge providers, subject experts, it not only has a lot of human involvement there, also including data, knowledge base and other technology of computer machine, which itself is a complex giant system. Therefore, the knowledge consulting attributed to the open complex giant system, the experts of HWME can be used to solve knowledge consulting problem. This paper proposes to use the user and experts of HWME to solve the problem of knowledge consulting in discussions while promoting improved knowledge base.

\section{RELATED THEORIES}

HWME is applied in many disciplines and fields to solve complex problems since it was proposed by Qian XueSen ${ }^{[6]}$. Zeng Zhenxiang ${ }^{[7]}$,Sha Jichang ${ }^{[8]}$,Wang Feiyue $^{[9]}$,Ni Ming ${ }^{[10]}$ respectively applying HWME to decision making, war, urban development, and enterprise information. HWME and information fusion techniques are combined to solve the problem of enterprise competitive intelligence system ${ }^{[1]}$.Hu Daiping puts that the knowledge visualization and HWME combination, offering a variety of models for the HWME experts to decision to improve efficiency ${ }^{[12]}$. HWME applied to knowledge organization and application. Liang Huiwen proposed the method of knowledge acquisition and application based on HWME networks, proposing a method to increase the rational of decision knowledge ${ }^{[13]}$.The HWME is applied to knowledge 
sharing, solving the problem of virtual team knowledge sharing ${ }^{[14]}$.HWME is applied to weapon system, and take advantage of the cloud model and evidence theory to solve consensus analysis issues ${ }^{[15]}$. Therefore, HWME in addition to be used the military, economic, enterprise information, it also can be applied to knowledge sharing, knowledge services and knowledge organization. The HWME also can be used to solve the knowledge consulting problems, it also can be implemented across regions or country expert knowledge among consulting, implement discussion to modify at the same time, until the user satisfaction and improve the efficiency and effective of the discussion.

\section{KNOWLEDGE CONSULTING SERVICES BUILDING} BASED ON HALL FOR WORKSHOP OF META SYNTHESIS

\section{A. Characteristics}

Understanding the theory of HWME and application, $\mathrm{t}$ he theory of HWME and knowledge consulting is combin ed to build a knowledge consulting system of human-com puter based on HWME.

\section{1) Human-computer combination}

Human-computer combination(human-computer inter action and human- computer selection), according to the $\mathrm{s}$ cientific and effective program to organize man, database $\mathrm{s}$, and ontology base and others knowledge, constituting a harmony personification and efficient of people machine integration virtual work system, integrated use of various external requirements resources, enable experts to maximi ze the contribution of their knowledge and capacity, as $\mathrm{w}$ ell as the use of user participation in discuss, to solve user problems and meet the demand of users.

\section{2) Integration of consulting discussion process}

As the main working mode, the discuss use the theory of comprehensive integration, experts and users using the knowledge base constantly modifying discussion, discuss process is a continuous learning and modifying rising spir al process, and ultimately to meet the needs of users and o btain satisfactory resolution, but also the process of the $\mathrm{kn}$ owledge accumulation and applications.

3) The emergence of collective intelligence.

In the knowledge consulting based on HWME, the par ticipation of consultant, experts and users can integrate $\mathrm{kn}$ owledge resources, promoting the emergence of group wi sdom.

\section{B.Model}

According to process of HWME and knowledge consulting to build knowledge consulting models, users will need to resolve the problem to the consultant, consultant to analyze the problem, and query related expert, they will need to consult the questions submitted to the experts, if host can found virtual expert and using virtual expert knowledge, if no found related of virtual expert that is needed looking for reality expert, user involvement in the knowledge consulting process at the same time, references to other scholars building model, knowledge consulting model based on the HWME as shown in Fig .1, promoting the development of knowledge consulting model building based on HWME.

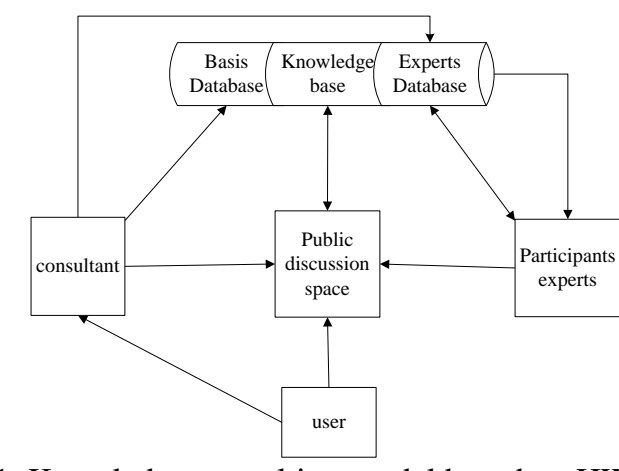

Figure 1. Knowledge consulting model based on HWME

This model has the following components: consultant, users, experts, the public discussion space and resources several major modules:

1) Consultant: Consultant includes virtual and real consultant. He accepts the issue of the users through interactions, and determines the themes of the consultation, and the theme refining into several major problems or keywords for discussion. Keywords can be classified as systems keywords, expert keywords, automatically keywords ${ }^{[16]}$.These keywords can be given some weight in order to determine the importance and order of keyword discuss. Meanwhile he contacts experts in relevant domains, organizes experts and user to discuss the issue.

2) User: In knowledge consulting based on HWME, the user is also involved in discussions and expresses their views and ideas. This can correct research direction and improve research efficiency.

3) Expert: Experts including real experts and virtual experts, the real expert have extensive knowledge; knowledge of virtual experts mainly refers to storage the knowledge of experts produced in discussion. Through the mutual-exchange of experts, user and consultant, Experts express their views in HMWE will have maximize and comprehensive expertise.

4) Resources base. In the HWME, in addition to participating in discussions, it should have some information and data as a reference for people's decisions. Resources main includes database, knowledge base (knowledge of past and existing knowledge, discussion knowledge, various related information, other knowledge) and expert database. In the HWME, neither experts nor the user in different places or even different countries can take advantage of these resources to express their views and opinions in the discussion.

5) Public discussion space. Public discussion space is the public places that experts, consultant and users to participate in discussion. It is a virtual public space, including discuss conducted between people and systems, experts and experts, users and experts, The experts and users can make adequate communication and interaction in it. It is a process of continuous exchange, inspire, share information each other and decision. The user and expert can work together to promote the emergence of collective wisdom. 


\section{A.Analysis of key technology}

In the knowledge consulting based on HWME, knowledge representation and visualization and retrieval are the key technology to achieve knowledge consulting and discussions as follows:

1) Knowledge representation. It is represented the human knowledge which exists in a different form the computer can be accepted and processed. It includes user's knowledge representation, knowledge base representation, expert's knowledge representation and the result knowledge representation four types in knowledge consulting based on HWME. This paper uses the way of ontology knowledge representation; ontology is the explicit formal specification of the shared conceptual model. It has a good conceptual hierarchy and logical reasoning ability. It not only has a detailed description to the concept, property, relationship, axiom and the constraint in the model, also can show its rich semantic relationship. The main purpose is to unify the concept of application areas and guarantee the semantic consistency.

2) The visualization process. Use the knowledge map to achieve visualize of discussion process, it can clearly showing the process of discussion, so that users, consultant and experts clear the discussion of key steps in which process to be modified. Ontology building knowledge map, it can overcome obstacles vary in different areas of environmental vocabulary, methods and tools generated to unifying concept in the application field, to achieve a certain level of knowledge sharing and reuse. After submitting user demand, the problem was refined by consultant and submitted to the experts and databases, user and knowledge base. The host will organize a comprehensive proposal to form a consolidated results, the results is tested by experts and user, if they are not satisfied with the test results, the results will be submitted to the host and discussed again until the users and expert are satisfied with the results.

3) Retrieval type. Retrieval type refers to the understanding of search engines and word of the operation ${ }^{[17]}$, with the search term operator to connect a computer to make the expressions constitute. Retrieval type can be analyzed to understand the structure and expression itself from retrieving two search terms. This paper used the technology of ontology retrieval. Ontology semantic model organization and annotation in knowledge base, ontology retrieval and knowledge retrieval support direct retrieval with natural language retrieval mode, this mode can increase keywords level rise to semantic, and the ontology have good support to the conceptual structure and logical reasoning, it also can avoid false, false detection, review and retrieval problems.

\section{CASE STUDY}

The impact of urbanization on the ecological environment is a typical complex problem, which has affected many aspects; knowledge of its consulting will reduce its negative impacts and promote ecological balanced development.

\section{A.Background}

Improve of the urbanization level is an important indicator of human civilization, but also the route one must take agricultural country to industrial country. With quickly development of urbanization, it has great promoted the development of economic and prosperity in rural areas. However, in the process of urbanization, people pay attention to the direct economic benefits, while ignoring the social and environmental benefits, The problems appeared in front of us, such as environment deteriorated, air pollution, water pollution, garbage siege, industrial pollution etc. The solution to these problems involves multiple interests, itself is a complex issue, so it can be solved based on knowledge consulting.

\section{A.Knowledge consulting process based on HWME}

According to consulting model based HWME, the influence of urbanization as a consulting task, building knowledge consulting process as shown the Fig .2.

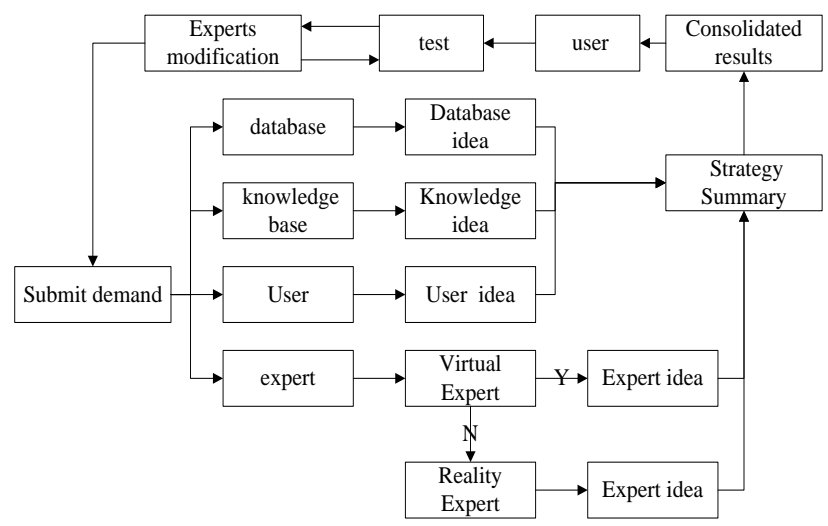

Figure 2. Knowledge consulting process visualization

Specific discuss process is as follows:

1) Clearly the tasks that need to be consulted. The task submitted to the consultant, the problem of urbanization affection is subdivided into several major keywords such as (urbanization and it affection, soil erosion, water ecological, industrial pollution and other city propose),determining the order of the discussion, the order of discussion is the key words shown in the above order.

2) Determines the scope of the problem and contact the relevant experts. Determine the scope of consultation is the impact of urbanization on ecological environment, using the database system to collect literature and expert information, and contacting relevant experts of urbanization and ecological environment, users to HWME. First is looking for the virtual expert search in the expert base, looking for experts ' views on whether there is a similar scheme and the knowledge used experts had discussed. If there have used knowledge to solve the current problems, such as other city have discussed in HMWE, we can use this knowledge. If there is not have the used knowledge, it is needs to contact reality expert. The reality experts and scholars to preliminary understand the problem, discuss scope main for the effects of urbanization, the first scope is the effects of urbanization, second specific is to found the related or similar 
experience of other city, finally to analyze the problem in specific city.

3) The expert discusses. Under the host guidance, host found related papers information in knowledge base, experts attending the meeting according to the discussion of key words order and give their opinions, that effects views of urbanization, users also participate in the discussion and express their views, reviewers and users to discuss solutions, consultant analysis the expert's knowledge, if experts exists differences or not satisfaction. Consultant is needed to redefine keywords, and then follow the steps to discuss until with the satisfied solutions; then follow the above steps to discuss until have satisfying result, and satisfactory result as information is contained in HWME.

4) Then initiate the next key problem will be discussed, it will have satisfactory results after multiparty interactive discussion again, and finally the impact of urbanization as a research object discussion. So far the entire problem has been discussed; the host summarized the whole issue sorting to determine the final result. If not satisfied, then the host considers view which needs to be re-discussed, if the results did not achieve the desired result, we need to modify the keywords or re-discus until satisfied. If satisfied, then output the final result to the user.

Through discussion, it not only to solve the user's problem, more importantly is comprehensive relevant expert opinions and user suggestions, which are integrated into the final result. It can enhance democracy and effectiveness of the proposed fully meet user needs. It is of great importance of the experts' tacit knowledge and it's explicit. Through the visualization of discussion process, recording tacit knowledge of users, experts and stored in the knowledge base, we can first select the tacit knowledge of virtual expert when the next similar knowledge consulting, related programs. If not found, it is looking for the reality experts to discuss.

\section{CONCLUSION}

This paper is from the perspective of knowledge consulting and HWME to consulting the platform based on HWME. In the support of HWME and multimedia technology, knowledge consulting platform using HWME can improve the efficiency of knowledge consulting. Through the construction of the knowledge base and knowledge map, knowledge consulting will be more clear and realization, using the wisdom of the Hall for workshop of meta-synthetic experts crowds not only can make users the necessary consultation results, and can also visualization present its own tacit knowledge, increasing knowledge consulting and improve the knowledge base for tacit knowledge share and development at the same time.

\section{ACKNOWLEDGMENT}

This work was financially supported by the Fundamental Research Funds for the Central Universities (2012B13814, 2013B09314)

\section{REFERENCES}

[1] Wang Tianni. Knowledge Consultation the Growth Points of Libraries' Knowledge Services in the Era of Big Data[J].Library \& Information, 2013 (2
[2] Ma Tianshu. Main Mode of University Library Knowledge Service [J].Research on Library Science,2013,(23).

[3] Zeng deyun. Characteristics model and promotion strategies of knowledge consulting services, $[\mathrm{J}]$. Journal of Library Science[J],2013,(7).

[4] Li Wenhui. Exploration on Knowledge Consultation [J].Work in Colleges and Universities, 2013,(6).

[5] LIUQING, WANG Gaibian. The Construction of Collaborative Reference Service System Based on the SECI Knowledge Creation Spiral-Taking Collaborative Reference Database of Japan as an Example[J].Library Tribune,2013,(5).

[6] QIAN Xuesen.A new discipline of science--The study of open complex giant system and its methodology [J].Urban Studies, 2005, 12(5):1-8.

[7] Zeng Zhenxiang. The Decision Support Center- the Development Trend of DSS [J].Systems Engineering and Electronics, 2000, (2).

[8] Sha Jichang, Mao Chilong, WU Yongbo, Chen Chao. The Study of the Technology for War Design Engineering [J].Systems Engineering-Theory \& Practice, 2005,(6).

[9]Wang Feiyue ,Dai Ruwei. A Complex System Approach for Studying Sustainable and Integrated Development of Metropolitan Transportation, Logistics and Ecosystems[J].Complex Systems and Complexity Science,2004,(2).

[10] Ni Ming,Zhang Bioresearch of Complex System for Implementing Enterprises Information Based on Hall for Workshop of MetaSynthetic Engineering[J].Library and Information Service,2009,(22).

[11] Song Xinping, Wu Xiaowei, Liu Jing. Competitive Intelligence System Based on a Hybrid Frame of Information Fusion and Hall for Workshop of Meta--Synthetic Engineering[J].Library and Information Service,2009,53(22):76-79.

[12] HU Daiping,WANG Huanchen. Building forecasting model system in workshop for hall of meta-synthetic engineering to support macro-economy decision[J].Journal of Systems Engineering, 2001, (5).

[13] LIANG Hui-wen,Wang Huimin. Method and Application of Integrated Discussion obtain knowledge -based networks[J].Journal of Southeast University (Philosophy and Social Science) ,2011,(2).

[14] Lin Xiang-yi,LUO Hong-yun,WU Hao,LI Li-ping. Knowledge Sharing Platform for Virtu-al Scientific Research Team Based on Hall for Workshop of Meta--Synthetic Engineering[J].Scientific Management Research,2012,(5).

[15] ZHOU Jian,ZHU Yaoqin.Tang Weiqing. Approach for analyzing consensus based on cloud model and evidence theory[J].Systems Engineering-Theory\&Practice,2012,(12).

[16] Zhou Shaoqi,Huang Xiyue. A model of a hall for a workshop of meta-synthetic engineering [J].Journal of Chongqing University (Natural Science Edition),, 2009,(6).

[17] Zeng Dejun, WANG Su-li. Design on Visualization System of Crisis Analysis Based on Experts Wisdom [J].Information Science, 2012, (9). 